\title{
Quantum dynamics via a time propagator in Wigner's phase space
}

\section{Grønager, Michael; Henriksen, Niels Engholm}

Published in:

Journal of Chemical Physics

Link to article, DOI:

$10.1063 / 1.469266$

Publication date:

1995

Document Version

Publisher's PDF, also known as Version of record

Link back to DTU Orbit

Citation (APA):

Grønager, M., \& Henriksen, N. E. (1995). Quantum dynamics via a time propagator in Wigner's phase space. Journal of Chemical Physics, 102(13), 5387-5395. https://doi.org/10.1063/1.469266

\section{General rights}

Copyright and moral rights for the publications made accessible in the public portal are retained by the authors and/or other copyright owners and it is a condition of accessing publications that users recognise and abide by the legal requirements associated with these rights.

- Users may download and print one copy of any publication from the public portal for the purpose of private study or research.

- You may not further distribute the material or use it for any profit-making activity or commercial gain

- You may freely distribute the URL identifying the publication in the public portal

If you believe that this document breaches copyright please contact us providing details, and we will remove access to the work immediately and investigate your claim. 


\title{
Quantum dynamics via a time propagator in Wigner's phase space
}

\author{
Michael Grønager and Niels Engholm Henriksen \\ Technical University of Denmark, Chemistry Department B, DTU-207, DK-2800 Lyngby, Denmark
}

(Received 2 November 1994; accepted 27 December 1994)

\begin{abstract}
We derive an expression for a short-time phase space propagator. We use it in a new propagation scheme and demonstrate that it works for a Morse potential. The propagation scheme is used to propagate classical distributions which do not obey the Heisenberg uncertainty principle. It is shown that the simple classical deterministic motion breaks down surprisingly fast in an anharmonic potential. Finally, we discuss the possibility of using the scheme as a useful approach to quantum dynamics in many dimensions. To that end we present a Monte Carlo integration scheme using the norm of the propagator as a part of the sampling function. (C) 1995 American Institute of Physics.
\end{abstract}

\section{INTRODUCTION}

The time evolution of quantum mechanical states is of central importance to many areas of chemistry and physics. The frontal attack on the time-dependent Schrödinger equation is one possibility. The numerical work in this approach scales, however, unfavorably with the dimensionality of the system. The use of a short-time propagator in conjunction with Monte Carlo integration is, at least in principle, the optimal approach. ${ }^{1}$ The advantage of this method is that the numerical work grows slowly with the dimensionality of the system. In standard formulations the propagator is a complex-valued object and a major problem in existing methods is that it is difficult to find a natural and good sampling function in the Monte Carlo integration schemes. In addition, the connection to classical pictures is not completely transparent. The propagator gives the amplitude for motion out of a given point in position space. A well-defined position implies that all momenta have the same weight and this situation never connects up with classical mechanics in an elementary fashion.

The purpose of the present paper is to suggest a new twist on existing propagation schemes as well as to provide some additional insight into the classical limit.

We take here as a starting point a propagator in the Wigner phase space formulation of quantum mechanics. ${ }^{2-5}$ This propagator gives the "pseudoprobability" for motion out of a specified point in phase space. The phase space propagator has some nice properties. It is real-valued and for systems which are at most harmonic the connection to classical mechanics is very transparent-the propagator is simply a delta distribution around the classical trajectory. The first property could make it a more convenient tool in conjunction with Monte Carlo integration and the second property gives a nice connection to the classical mechanics, on which our physical intuition is based.

This paper is organized in the following way: We begin by introducing a time propagator in phase space. We derive a general expression for a short-time propagator and develop an analytical expression for the Morse potential. Using this propagator repeatedly on a grid we consider the phase space quantum dynamics of a Morse oscillator. We present subsequently some work on a Monte Carlo integration scheme using the norm of the propagator as a part of the sampling function. Finally, the results are summarized and we consider what needs to be done in order to make the present scheme a versatile tool for quantum dynamics in many dimensions.

\section{THEORY}

First we should note that all derivations throughout this paper are based on a one dimensional system, however generalization to many dimensions is straightforward.

Let $\hat{A}(t)$ denote a quantum mechanical operator, the corresponding Wigner phase space function, is now obtained performing the Wigner transform ${ }^{2,3}$

$$
A(q, p, t)=\frac{1}{2 \pi \hbar} \int d \eta e^{-(i / \hbar) p \eta}\left\langle q-\frac{\eta}{2}|\hat{A}(t)| q+\frac{\eta}{2}\right\rangle .
$$

The Wigner phase space density function $f_{\psi}(q, p, t)$ is obtained transforming the density operator $\hat{\rho}(t)=|\psi(t)\rangle\langle\psi(t)|$. From which it follows that

$$
\int d q \int d p f_{\psi}(q, p, t)=1 \text {. }
$$

The time evolution is governed by ${ }^{3}$

$$
\frac{\partial}{\partial t} f_{\psi}(q, p, t)=\left[H(q, p), f_{\psi}(q, p, t)\right]_{\text {Moyal }},
$$

where the Moyal-bracket is defined by

$$
\begin{aligned}
{[A, B]_{\text {Moyal }} \equiv } & \frac{2}{\hbar} \sin \left[\frac{\hbar}{2}\left(\frac{\partial}{\partial q_{A}} \frac{\partial}{\partial p_{B}}-\frac{\partial}{\partial q_{B}} \frac{\partial}{\partial p_{A}}\right)\right] \\
& \times A(q, p, t) B(q, p, t),
\end{aligned}
$$

where the subscripts $A$ and $B$ indicate that the operator acts only on $A(q, p, t)$ and $B(q, p, t)$, respectively.

\section{A. The phase space propagator}

Following McLafferty ${ }^{4}$ we define a propagator in phase space $K(2,1)=K\left(q_{2}, p_{2}, t_{2} ; q_{1}, p_{1}, t_{1}\right)$ by the property: Any phase space density function can be obtained as the convolution

$$
\begin{aligned}
f_{\psi}\left(q_{2}, p_{2}, t_{2}\right)= & \int d q_{1} \int d p_{1} K\left(q_{2}, p_{2}, t_{2} ; q_{1}, p_{1}, t_{1}\right) \\
& \times f_{\psi}\left(q_{1}, p_{1}, t_{1}\right) .
\end{aligned}
$$

We can of course then write $f_{\psi}\left(q_{1}, p_{1}, t_{1}\right)$ as a similar convolution, and can hence identify that the propagator satisfies 


$$
\begin{aligned}
& K\left(q_{2}, p_{2}, t_{2} ; q_{0}, p_{0}, t_{0}\right) \\
& =\int d q_{1} \int d p_{1} K\left(q_{2}, p_{2}, t_{2} ; q_{1}, p_{1}, t_{1}\right) \\
& \quad \times K\left(q_{1}, p_{1}, t_{1} ; q_{0}, p_{0}, t_{0}\right) .
\end{aligned}
$$

Inserting the definition (5) into Eq. (3) we get that

$$
\begin{aligned}
\frac{\partial}{\partial t_{2}} \int d q_{1} \int d p_{1} K\left(q_{2}, p_{2}, t_{2} ; q_{1}, p_{1}, t_{1}\right) f_{\psi}\left(q_{1}, p_{1}, t_{1}\right) \\
=\left[H\left(q_{2}, p_{2}\right), \int d q_{1} \int d p_{1}\right. \\
\left.\quad \times K\left(q_{2}, p_{2}, t_{2} ; q_{1}, p_{1}, t_{1}\right) f_{\psi}\left(q_{1}, p_{1}, t_{1}\right)\right]_{\text {Moyal }} .
\end{aligned}
$$

And since $f_{\psi}\left(q_{1}, p_{1}, t_{1}\right)$ can be chosen arbitrarily we get

$$
\begin{aligned}
\frac{\partial}{\partial t_{2}} & K\left(q_{2}, p_{2}, t_{2} ; q_{1}, p_{1}, t_{1}\right) \\
& =\left[H\left(q_{2}, p_{2}\right), K\left(q_{2}, p_{2}, t_{2} ; q_{1}, p_{1}, t_{1}\right)\right]_{\text {Moyal }} .
\end{aligned}
$$

Finally we get, setting $t_{2}=t_{1}$ in Eq. (5), that

$$
K\left(q_{2}, p_{2}, t_{1} ; q_{1}, p_{1}, t_{1}\right)=\delta\left(q_{2}-q_{1}\right) \delta\left(p_{2}-p_{1}\right) .
$$

Equation (8) can be solved analytically for systems which are at most harmonic. In this case the Moyal-bracket reduces to the Poisson-bracket and we get that every point follows the classical trajectory, hence we can write for the propagator $^{5}$

$$
\begin{aligned}
K\left(q_{2}, p_{2}, t_{2} ; q_{1}, p_{1}, t_{1}\right)= & \delta\left[q_{2}\left(q_{1}, p_{1} ; t_{2}-t_{1}\right)-q_{1}\right] \\
& \times \delta\left[p_{2}\left(q_{1}, p_{1} ; t_{2}-t_{1}\right)-p_{1}\right],
\end{aligned}
$$

where the notation $\left(q_{1}, p_{1} ; t_{2}-t_{1}\right)$ means propagated along the classical trajectory from $t_{1}$ to $t_{2}$ starting in $\left(q_{1}, p_{1}\right)$. This simple form for the propagator is the inspiration for the so called "Wigner method" for time propagation. ${ }^{6-8}$ Here it is assumed that this propagator is a good approximation for not too anharmonic systems.

The short-time propagator we get using Eq. (8) for the first order time derivative. Retaining terms only up to first order in a Taylor expansion, we get

$$
\begin{aligned}
& K\left(q_{2}, p_{2}, \Delta t ; q_{1}, p_{1}, 0\right) \\
&= K\left(q_{2}, p_{2}, 0 ; q_{1}, p_{1}, 0\right) \\
&+\Delta t\left[H\left(q_{2}, p_{2}\right), K\left(q_{2}, p_{2}, 0 ; q_{1}, p_{1}, 0\right)\right]_{\text {Moyal }} .
\end{aligned}
$$

We now use Eq. (9), but for the delta distributions we use the functional form

$$
\delta\left(q_{2}-q_{1}\right)=\frac{1}{2 \pi \hbar} \int d p^{\prime} e^{(i / \hbar)\left(q_{2}-q_{1}\right) p^{\prime}},
$$

which yields

$$
\begin{aligned}
K(2,1)= & \frac{1}{(2 \pi \hbar)^{2}} \int d q^{\prime} \int d p^{\prime}\left\{e^{(i / \hbar)\left(q_{2}-q_{1}\right) p^{\prime}}\right. \\
& \times e^{(i / \hbar)\left(p_{2}-p_{1}\right) q^{\prime}} \\
& +\Delta t\left[H\left(q_{2}, p_{2}\right), e^{(i / \hbar)\left(q_{2}-q_{1}\right) p^{\prime}}\right. \\
& \left.\times e^{(i / \hbar)\left(p_{2}-p_{1}\right) q^{\prime}}\right]_{\text {Moyal }\} .}
\end{aligned}
$$

Evaluating the Moyal bracket and defining

$$
\begin{aligned}
& \tilde{H}=\tilde{T}+\tilde{V}, \quad \tilde{T}=T\left(p_{2}+\frac{p^{\prime}}{2}\right)-T\left(p_{2}-\frac{p^{\prime}}{2}\right), \\
& \tilde{V}=V\left(q_{2}-\frac{q^{\prime}}{2}\right)-V\left(q_{2}+\frac{q^{\prime}}{2}\right) .
\end{aligned}
$$

We get for the short-time propagator,

$$
\begin{aligned}
K(2,1)= & \frac{1}{(2 \pi \hbar)^{2}} \int d q^{\prime} \int d p^{\prime}\left(1-\Delta t \frac{i}{\hbar} \tilde{H}\right) \\
& \times e^{(i / \hbar)\left(q_{2}-q_{1}\right) p^{\prime}} e^{(i / \hbar)\left(p_{2}-p_{1}\right) q^{\prime}} .
\end{aligned}
$$

We now split up the integrals in a kinetic and a potential part

$$
\begin{aligned}
K(2,1)= & \frac{1}{2 \pi \hbar} \int d p^{\prime}\left(1-\Delta t \frac{i}{\hbar} \tilde{T}\right) e^{(i / \hbar)\left(q_{2}-q_{1}\right) p^{\prime}} \\
& \times \frac{1}{2 \pi \hbar} \int d q^{\prime}\left(1-\Delta t \frac{i}{\hbar} \tilde{V}\right) e^{(i / \hbar)\left(p_{2}-p_{1}\right) q^{\prime}} \\
\equiv & I\left(q_{2}-q_{1}, p_{2}\right) J\left(p_{2}-p_{1}, q_{2}\right),
\end{aligned}
$$

where we have used that to first order in $\Delta t$ is

$$
1+\frac{i \Delta t}{\hbar} \tilde{H}=\left(1+\frac{i \Delta t}{\hbar} \tilde{T}\right)\left(1+\frac{i \Delta t}{\hbar} \tilde{V}\right) .
$$

Since $T(p)=p^{2} / 2 m$ we get $\tilde{T}=p^{\prime} p_{2} / m$, using for $I\left(q_{2}-q_{1}, p_{2}\right)$ the first order approximation $1-x=e^{-x}$, we get

$$
\begin{aligned}
I\left(q_{2}-q_{1}, p_{2}\right) & =\frac{1}{2 \pi \hbar} \int d p^{\prime} e^{(i / \hbar)\left[\left(q_{2}-q_{1}\right)-(\Delta t / m) p_{2}\right] p^{\prime}} \\
& =\delta\left(q_{2}-q_{1}-\frac{\Delta t}{m} p_{2}\right) .
\end{aligned}
$$

Our final expression for the propagator is then

$$
K(2,1)=\delta\left(q_{2}-q_{1}-\frac{\Delta t}{m} p_{2}\right) J\left(p_{2}-p_{1}, q_{2}\right) .
$$

Finally we can assure us that $K(2,1)$ is in fact a real function. $I\left(q_{2}-q_{1}, p_{2}\right)$ is certainly real, and $J\left(p_{2}-p_{1}, q_{2}\right)$ is formed from a Fourier transform of a function which has an even real and an odd imaginary part, meaning that the imaginary part cancels out due to symmetry.

\section{B. A short-time propagator for the Morse oscillator}

For the Morse oscillator the potential is given by

$V(q)=D_{e}\left(1-e^{-\beta_{q}}\right)^{2}$. 
We change to dimensionless variables; $\tau=(\Delta t / \hbar) D_{e}$, $Q=\beta q_{2}$ and $\Delta P=P_{2}-P_{1}=\left(p_{2}-p_{1}\right) / \beta \hbar$, and get for the $J\left(P_{2}-P_{1}, Q_{2}\right)$ integral, using the first order approximation $1-x=1 /(1+x)$,

$$
\begin{aligned}
\hbar \beta J= & \frac{1}{2 \pi} \int d Q^{\prime} \\
& \times \frac{e^{i Q^{\prime} \Delta P}}{1+i \tau e^{-Q}\left[e^{-Q}\left(e^{Q^{\prime}}-e^{-Q^{\prime}}\right)-2\left(e^{1 / 2 Q^{\prime}}-e^{-1 / 2 Q^{\prime}}\right)\right]}
\end{aligned}
$$

using that $e^{x}-e^{-x}=-2 i \sin (i x)$ we get

$$
\begin{aligned}
\hbar \beta J= & \frac{1}{2 \pi} \int d Q^{\prime} \\
& \times \frac{e^{i Q^{\prime} \Delta P}}{1+2 \tau e^{-Q}\left[e^{-Q} \sin \left(i Q^{\prime}\right)-2 \sin \left(\frac{1}{2} i Q^{\prime}\right)\right]} .
\end{aligned}
$$

Using the residue theorem and Jordan's lemma, for $\Delta P>0$, we can write this integral as [for simplicity we write $f\left(Q^{\prime}\right)$ for the integrand],

$$
\hbar \beta J=i \sum_{k} \operatorname{Res}\left[f\left(Q^{\prime}\right) ; Q_{k}^{\prime}\right]
$$

where we sum over $Q_{k}^{\prime}$ in the upper complex plane only. The values of $Q_{k}^{\prime}$ are found solving the equation

$$
1+2 \tau e^{-Q}\left[e^{-Q} \sin \left(i Q^{\prime}\right)-2 \sin \left(\frac{1}{2} i Q^{\prime}\right)\right]=0 .
$$

Substituting $Q^{\prime}=i \Theta$ we see that if $\Theta$ is a solution to Eq. (24) so is $\Theta^{*}$,

$$
\begin{aligned}
& 0=1-2 \tau e^{-Q}\left(e^{-Q} \sin \Theta *-2 \sin \frac{1}{2} \Theta *\right) \Leftrightarrow \\
& 0=\left[1-2 \tau e^{-Q}\left(e^{-Q} \sin \Theta-2 \sin \frac{1}{2} \Theta\right)\right]^{*} \Leftrightarrow \\
& \left.0=1-2 \tau e^{-Q}\left(e^{-Q} \sin \Theta-2 \sin \frac{1}{2} \Theta\right)\right] .
\end{aligned}
$$

Meaning that the solutions are either real or complex conjugate pairs. Further we see that provided $\Theta$ is a solution, then due to the periodic nature of the sin-function, so is $\Theta \pm 4 \pi$. Finally we use the quartic nature of the denominator of Eq. (21) to order the solutions; in the substitution $x$ $=e^{(1 / 2) Q^{\prime}}=e^{(1 / 2) i \Theta}$ the denominator of Eq. (21) can be written

$$
\begin{aligned}
& 1+i \tau e^{-Q}\left[e^{-Q}\left(e^{Q^{\prime}}-e^{-Q^{\prime}}\right)-2\left(e^{(1 / 2) Q^{\prime}}-e^{-(1 / 2) Q^{\prime}}\right)\right] \\
& =i \tau e^{-2 Q_{x}-2}\left(x^{4}-2 e^{Q} x^{3}-i \frac{e^{2 Q}}{\tau} x^{2}+2 e^{Q} x-1\right) .
\end{aligned}
$$

There will hence be four fundamentally different solutions $x_{j}, j=1,2,3,4$ each resulting in an infinity of equally spaced solutions $\Theta_{j}+4 \pi k, k=0, \pm 1, \pm 2, \ldots$. We choose $\Theta_{j}$ to have a real part in $\left[0 ; 4 \pi\left[\right.\right.$. In terms of $Q^{\prime}$ we now get

$$
\begin{aligned}
& Q^{\prime}=Q_{j k}^{\prime}=i\left(\Theta_{j}+4 \pi k\right), \quad \operatorname{Im}\left[Q_{j}\right] \in[0 ; 4 \pi[, \\
& j=1,2,3,4, \quad k=0, \pm 1, \pm 2, \ldots .
\end{aligned}
$$

Now the residues in the upper complex plane are those with $k=0,1,2, \ldots$ and the integral reduces to

$$
\hbar \beta J=i \sum_{j=1}^{4} \sum_{k=0}^{\infty} \operatorname{Res}\left[f\left(Q^{\prime}\right) ; Q_{j k}\right]=i \sum_{j=1}^{4} \sum_{k=0}^{\infty} \lim _{Q^{\prime} \rightarrow Q_{j k}^{\prime}} \frac{\left(Q^{\prime}-Q_{j k}^{\prime}\right) e^{i Q^{\prime} \Delta P}}{1+2 \tau e^{-Q}\left[e^{-Q} \sin \left(i Q^{\prime}\right)-2 \sin \left(\frac{1}{2} i Q^{\prime}\right)\right]} .
$$

Expanding the denominator to first order around $Q_{j k}^{\prime}$, we get a zero for the zeroth-order term, since $Q_{j k}^{\prime}$ are all roots in the denominator,

$$
\hbar \beta J=i \sum_{j=1}^{4} \sum_{k=0}^{\infty} \lim _{Q^{\prime} \rightarrow Q_{j k}^{\prime}} \frac{\left(Q^{\prime}-Q_{j k}^{\prime}\right) e^{i Q^{\prime} \Delta P}}{2 i \tau e^{-Q}\left[e^{-Q} \cos \left(i Q_{j k}^{\prime}\right)-\cos \left(\frac{1}{2} i Q_{j k}^{\prime}\right)\right]\left(Q^{\prime}-Q_{j k}^{\prime}\right)}=\frac{e^{Q}}{2 \tau} \sum_{j=1}^{4} \sum_{k=0}^{\infty} \frac{e^{i Q_{j k}^{\prime} \Delta P}}{e^{-Q} \cos \left(i Q_{j k}^{\prime}\right)-\cos \left(\frac{1}{2} i Q_{j k}^{\prime}\right)} .
$$

We now insert $i\left(\Theta_{j}+4 \pi k\right)$ for $Q_{j k}^{\prime}$ and get

$$
\hbar \beta J=\frac{e^{Q}}{2 \tau} \sum_{j=1}^{4} \frac{e^{-\Theta_{j} \Delta P}}{e^{-Q} \cos \Theta_{j}-\cos \frac{1}{2} \Theta_{j}} \sum_{k=0}^{\infty} e^{-4 \pi \Delta P k}
$$

since $\Delta P>0$ we can sum the geometric series, and hence we get

$$
\hbar \beta J=\frac{e^{Q}}{2 \tau} \frac{1}{1-e^{-4 \pi \Delta P}} \sum_{j=1}^{4} \frac{e^{-\Theta_{j} \Delta P}}{e^{-Q} \cos \Theta_{j}-\cos \frac{1}{2} \Theta_{j}} .
$$

Using the fact that $J$ is analytical ensures that it is also valid for $\Delta P \leqslant 0$. Further it ensures that since $\Theta_{j}$ are either real or complex conjugate pairs $\hbar \beta J$ will indeed be real. 


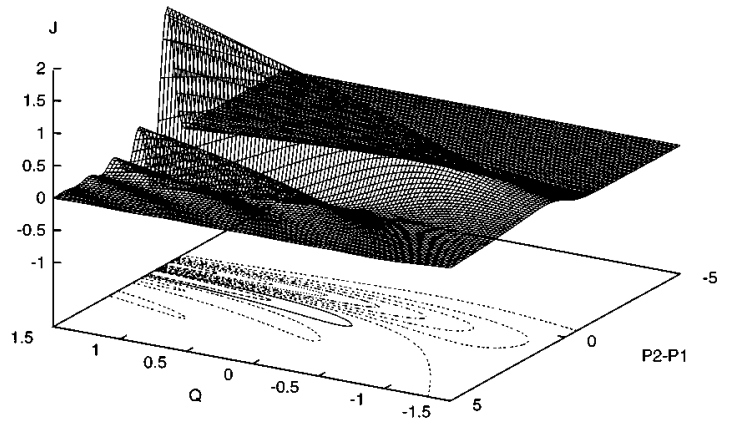

FIG. 1. A plot of the potential part of the short-time propagator for the Morse oscillator; $\hbar \beta J$ vs $Q$ and $P_{2}-P_{1}$, with $\tau=0.1$.

To compute the value of $\hbar \beta J$ we first solve the quartic equation

$$
x^{4}-2 e^{Q} x^{3}-i \frac{e^{2 Q}}{\tau} x^{2}+2 e^{Q} x-1=0
$$

using a standard formula (Decartes-Euler or Ferrari). ${ }^{9}$ Then we find the $\Theta_{j}$ from

$$
\Theta_{j}=-2 i \ln x_{j}
$$

(where the argument of $\ln$ is in the interval ]0,2 $\pi]$ ) $\hbar \beta J$ is now computed by insertion in Eq. (31). In Fig. $1 \hbar \beta J$ is plotted as a function of $Q$ and $\Delta P$. It is certainly a much more complicated function than the potential part of the propagator for the harmonic oscillator.

\section{RESULTS AND DISCUSSION}

\section{A. A finite grid propagation scheme}

We shall now introduce a new propagation scheme based on the phase space propagator. For a short time propagation we get from Eqs. (5) and (19),

$$
\begin{aligned}
f_{\psi}\left(q_{2}, p_{2}, \Delta t\right)= & \int d q_{1} \int d p_{1} \delta\left(q_{2}-q_{1}-\frac{\Delta t}{m} p_{2}\right) \\
& \times J\left(p_{2}-p_{1}, q_{2}\right) f_{\psi}\left(q_{1}, p_{1}, 0\right)
\end{aligned}
$$

we can easily integrate over $q_{1}$ and get

$$
\begin{aligned}
f_{\psi}\left(q_{2}, p_{2}, \Delta t\right)= & \int d p_{1} J\left(p_{2}-p_{1}, q_{2}\right) \\
& \times f_{\psi}\left(q_{2}-\frac{\Delta t}{m} p_{2}, p_{1}, 0\right) .
\end{aligned}
$$

If we now place $f_{\psi}$ on a finite grid, choosing a second order approximation for values outside the grid points

$$
\begin{aligned}
f_{\psi}\left(q_{i}+\Delta, p_{j}, 0\right)= & \frac{1}{2} \Delta(\Delta-1) f_{\psi}\left(q_{i-1}, p_{j}, 0\right) \\
& +\left(1-\Delta^{2}\right) f_{\psi}\left(q_{i}, p_{j}, 0\right) \\
& +\frac{1}{2} \Delta(\Delta+1) f_{\psi}\left(q_{i+1}, p_{j}, 0\right)
\end{aligned}
$$

the propagation scheme is obvious.

We start out by testing the validity of this scheme, and for this purpose we propagate a minimum uncertainty state (MUS),

$$
f_{\mathrm{MUS}}(Q, P, 0)=\frac{1}{\pi} e^{-\left[\sigma^{-2}\left(Q-Q_{0}\right)^{2}+\sigma^{2}\left(P-P_{0}\right)^{2}\right]} .
$$

$Q$ and $P$ are in the same dimensionless units as we defined earlier and $\sigma=\sqrt{2} \Delta Q=(\sqrt{2} \Delta P)^{-1}$, i.e., $\Delta Q \Delta P=\frac{1}{2}$. We choose $\sigma=1 / \sqrt{\lambda}, P_{0}=0$ and $Q_{0}=\sqrt{2 / \lambda}$ as in Ref. 7 . Note that the Morse oscillator is fully described by the dimensionless parameter $\lambda=\sqrt{2 m D_{e}} / \beta \hbar .{ }^{10}$ In the same dimensionless units the propagation scheme becomes

$$
\begin{aligned}
f_{\psi}\left(Q_{2}, P_{2}, \tau\right)= & \int d P_{1} \hbar \beta J\left(P_{2}-P_{1}, Q_{2}\right) \\
& \times f_{\psi}\left(Q_{2}-\frac{2 \tau}{\lambda^{2}} P_{2}, P_{1}, 0\right) .
\end{aligned}
$$

Using this repeatedly we are able to propagate the minimum uncertainty state a tour around in phase space. In Fig. 2 the motion is plotted as snapshots. Corresponding values of norm, overlap to the 0 th, 1 st, and 2 nd eigenstates ${ }^{10}$ and the energy are shown in Table I. We see that all these check values are indeed constant to within a percent. An arbitrary precision can be obtained making the grid bigger and the time steps smaller, for this calculation the grid consisted of $80 \times 80$ points and the time steps was $\tau=0.1$. From this calculation we see that the scheme works. It is also clear from the plots that the propagation is not entirely classical, since areas of new amplitudes form and vanish, as pointed out in

TABLE I. Check values of the minimum uncertainty state propagation, plotted in Fig. 2.

\begin{tabular}{rccccc}
\hline \hline$\tau$ & $\int d Q \int d P f(\tau)$ & $\int d Q \int d P H f(\tau)$ & $2 \pi \int d Q \int d P f_{0} f(\tau)$ & $2 \pi \int d Q \int d P f_{1} f(\tau)$ & $2 \pi \int d Q \int d P f_{2} f(\tau)$ \\
\hline 0 & 1.000 & 0.107 & 0.475 & 0.438 & 0.438 \\
10 & 1.000 & 0.108 & 0.471 & 0.436 & 0.085 \\
20 & 0.999 & 0.108 & 0.470 & 0.431 & 0.090 \\
30 & 0.999 & 0.109 & 0.472 & 0.429 & 0.091 \\
40 & 0.999 & 0.110 & 0.471 & 0.431 & 0.429 \\
50 & 0.999 & 0.110 & 0.469 & 0.472 & 0.098 \\
60 & 0.999 & 0.110 & 0.471 & 0.086 \\
70 & 0.999 & 0.110 & &
\end{tabular}



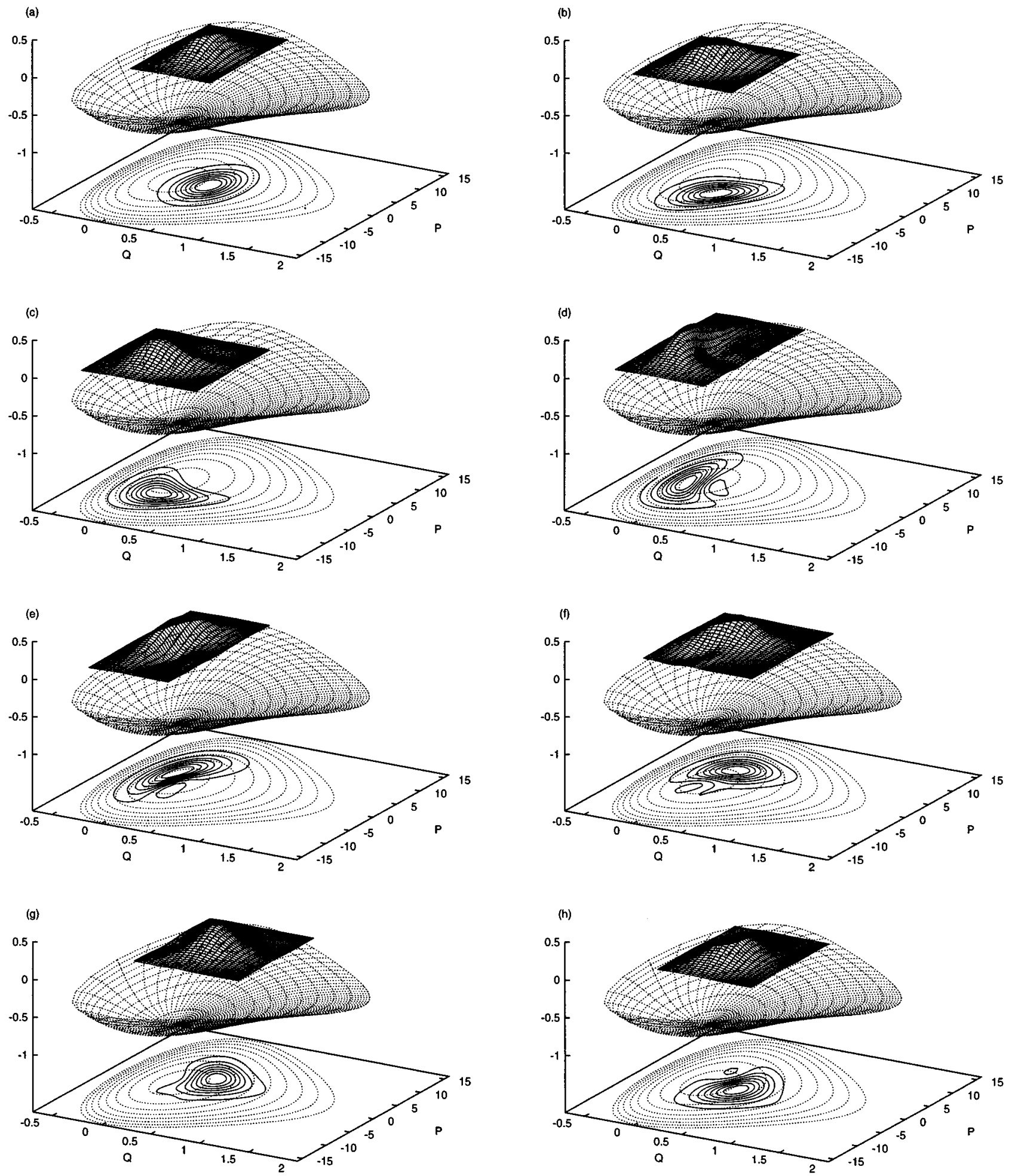

FIG. 2. (a),(b),..,(h) corresponds to the minimum uncertainty state being propagated to, respectively, $\tau=0,10, \ldots, 70$ in a Morse potential with $\lambda=20$. Contours for the potential are taken as the first eight energy eigenvalues, contours for the Wigner function are $-0.2,0.2, \ldots, 0.26$. We see that even though the center of the distribution essentially follows a classical propagation, some of the distribution takes negative values during the propagation, and hence the classical picture breaks down

Ref. 11. Hence we cannot describe the motion as the classical motion of phase space points. This is in accordance with the results of Ref. 7.

We shall now try to make a smooth connection to classical mechanics. With the propagation scheme introduced we are in fact able to propagate any distribution, even though it does not satisfy the Heisenberg uncertainty principle. Take that we know the exact position and momentum of a particle to a certain time, i.e., the distribution arising is the delta distribution 


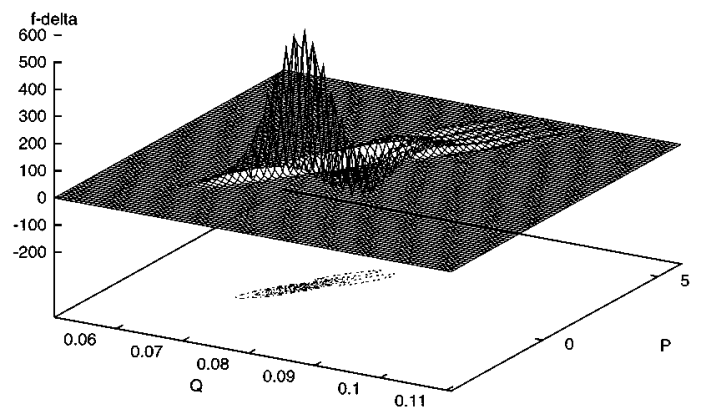

FIG. 3. This plot shows the form of a delta distribution propagated up to $\tau=0.2$ in a Morse potential with $\lambda=20$. The delta distribution was initially centered at $Q_{0}=\sqrt{2} / \lambda, P_{0}=0$. We see that the classical picture breaks down immediately after the propagation is started.

$$
f_{\delta}\left(Q_{1}, P_{1}, 0\right)=\delta\left(Q_{1}-Q_{0}\right) \delta\left(P_{1}-P_{0}\right)
$$

This we can plug into Eq. (38), integrating twice, and hence obtain the analytical form of the delta function after two small time steps,

$$
\begin{aligned}
f_{\delta}\left(Q_{3}, P_{3}, 2 \tau\right) & \\
= & \frac{\lambda^{2}}{2 \tau} \hbar \beta J\left[Q_{3}, 2 P_{3}-\frac{\lambda^{2}}{2 \tau}\left(Q_{3}-Q_{0}\right), \tau\right] \\
& \times \hbar \beta J\left[Q_{3}-\frac{2 \tau}{\lambda^{2}}, \frac{\lambda^{2}}{2 \tau}\left(Q_{3}-Q_{0}\right)-P_{3}-P_{0}, \tau\right] .
\end{aligned}
$$

This function is plotted in Fig. 3 from which it is seen that even though the distribution is narrow it is certainly no longer a delta distribution. This in fact enables us to put it on a grid and propagate it further using our propagation scheme. However since it is still quite narrow the grid would have to be very fine-meshed, and since the superposition of the delta distribution on the eigenstates of the Morse oscillator contains considerable contributions from high energy states the grid would also have to be quite large in order to represent the time evolution properly. All in all this would require a grid containing many points, making the calculation almost impossible. With the biggest grids we were working on (300 $\times 300$ points) we only succeeded to propagate the delta distribution up to $\tau \sim 3$. In order to show what is happening, when we propagate a delta distribution, we instead propagated another narrow-non-Heisenberg-distribution; a squeezed Gaussian,

$$
f_{\gamma}(Q, P, 0)=\frac{\gamma}{\pi} e^{-\gamma\left[\sigma^{-2}\left(Q-Q_{0}\right)^{2}+\sigma^{2}\left(P-P_{0}\right)^{2}\right]}
$$

with $\gamma=10$ and $\sigma, Q_{0}$ and $P_{0}$ as before. The uncertainty of this state can easily be calculated to be $\Delta Q \Delta P=1 /(2 \gamma)$, and hence is Heisenberg's uncertainty principle violated by a factor of 10. The propagation is shown for $\lambda=20$ in Fig. 4 and for $\lambda=120$ in Fig. 5. For $\lambda=20$ the propagation is highly nonclassical, however as we increase $\lambda$ to 120 the distribution only feels the harmonic part of the potential and hence behaves more classical as we would expect from the form of the time propagator for the harmonic oscillator [Eq. (10)].

The calculation illustrates that the concept of classical deterministic motion breaks down surprisingly fast in an anharmonic potential. A comparison between Figs. 2 and 4 shows, however, that the overall motion of the quantum mechanical phase space distribution is much more classical than suggested by the motion of individual phase space points (narrow distributions).

\section{B. Monte Carlo integration}

It is natural to mention Monte Carlo integration when discussing propagation via a propagator. The problem in using Monte Carlo techniques in ordinary Feynman path integration lies in the lack of a good sampling function, this is because the propagators are complex with a norm independent of the integration variables. Usually this is dealt with by introducing some other sampling function based on some physical knowledge of the system, e.g., a Gaussian distribution around the classical path. ${ }^{1}$ This however, will not always assure us fast convergence; what if the system does not behave classical at all? If we instead use the phase space propagator, we are assured the fastest convergence we could possible hope for; ${ }^{12}$ the phase space propagator has no complex oscillations and the norm dependents strongly on the integration variables. It is hence obvious to use the norm of the propagator as a part of the sampling function in a Monte Carlo calculation.

We considered especially the calculation of the quantity

$$
\begin{aligned}
P_{\phi}(t) & =|\langle\phi \mid \psi(t)\rangle|^{2} \\
& =2 \pi \hbar \int d q \int d p f_{\phi}(q, p) f_{\psi}(q, p, t) .
\end{aligned}
$$

This we can compute using Eq. (5) followed by repeated use of Eq. (6),

$$
\begin{aligned}
P_{\phi}(t)= & 2 \pi \hbar \int d q_{N} \int d p_{N} \int d q_{N-1} \int d p_{N-1} \cdots \\
& \times \int d q_{0} \int d p_{0} f_{\phi}\left(q_{N}, p_{N}\right) \times K(N, N-1) \cdots \\
& \times K(1,0) f_{\psi}\left(q_{0}, p_{0}, t\right)
\end{aligned}
$$

We now use Eqs. (16) and (18) to do all but one of the position integrals, leaving the expression 
(a)

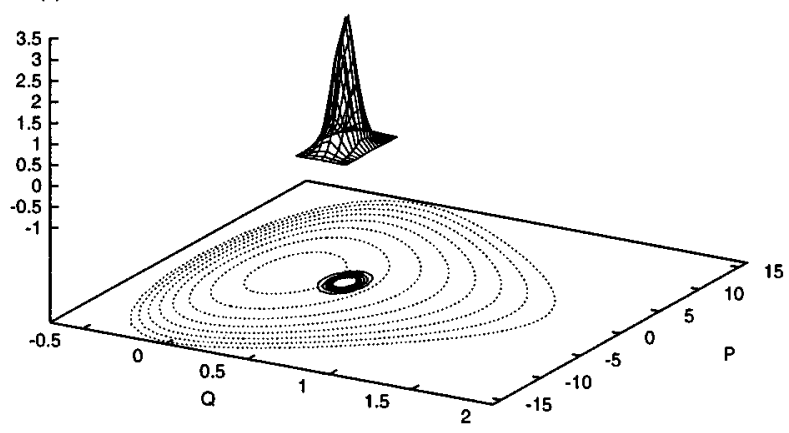

(c)

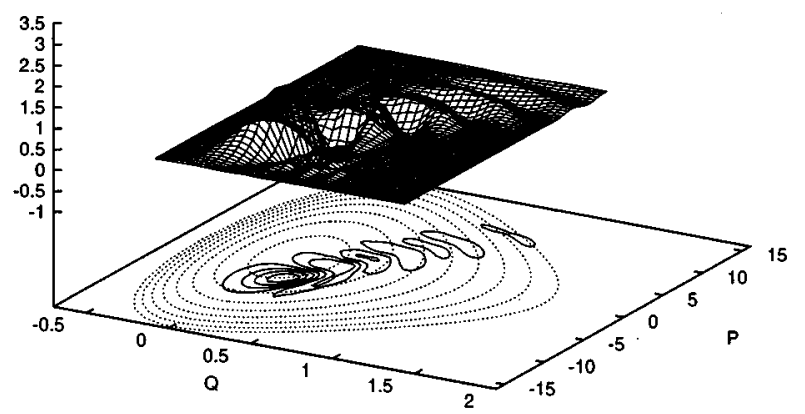

(e)

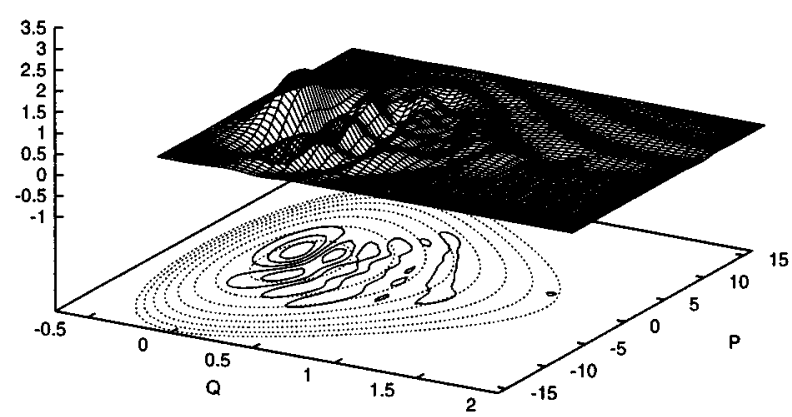

(g)

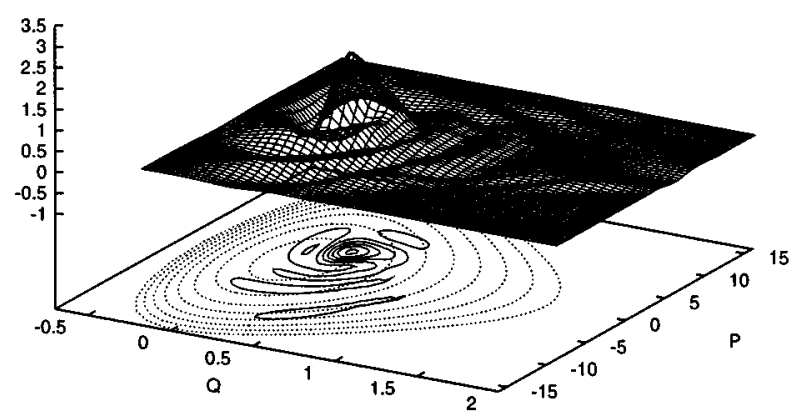

(b)

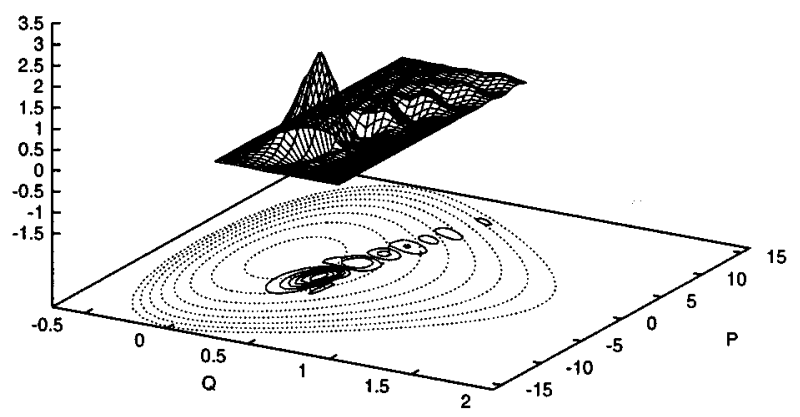

(d)

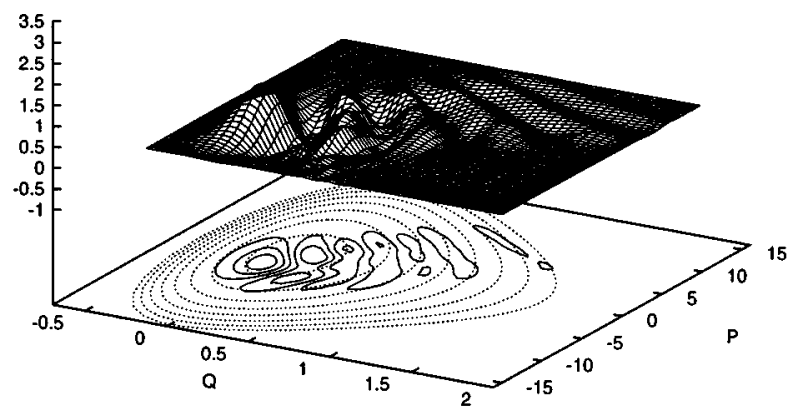

(f)

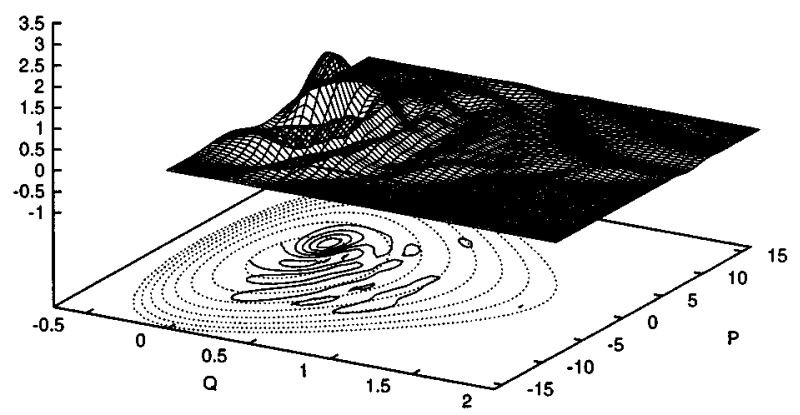

(h)

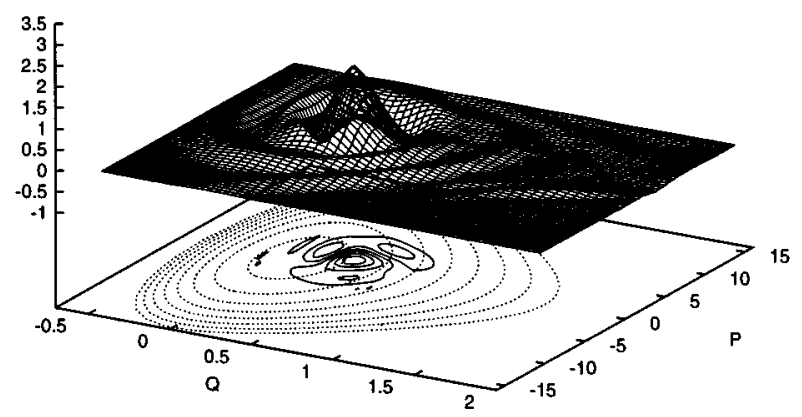

FIG. 4. (a),(b),..,(h) corresponds to the squeezed Gaussian being propagated in a Morse potential with $\lambda=20$, up to, respectively, $\tau=0$, $10, \ldots, 70$. Contours for the potential are taken as the first eight energy eigenvalues, contours for the Wigner function are $-0.4,-0.1, \ldots, 1.7$.

$$
\begin{aligned}
P_{\phi}(t)= & 2 \pi \hbar \int d q_{N} \int d p_{N} \int d p_{N-1} \cdots \\
& \times \int d p_{0} f_{\phi}\left(q_{N}, p_{N}\right) \times J\left(p_{N}-p_{N-1}, q_{N}\right) \cdots \\
& \times J\left(p_{1}-p_{0}, q_{1}\right) f_{\psi}\left(q_{0}, p_{0}, 0\right)
\end{aligned}
$$

with the additional condition

$$
q_{i}=q_{i+1}-\frac{\Delta t}{m} p_{i+1}, \quad i=0,1, \ldots, N-1 .
$$

This integral we can solve using Monte Carlo integration, sampling after the Metropolis scheme. ${ }^{13,12}$ First we define 
(a)

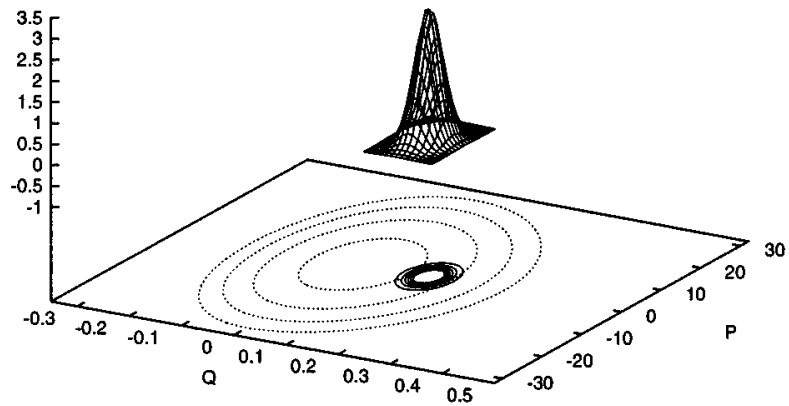

(c)

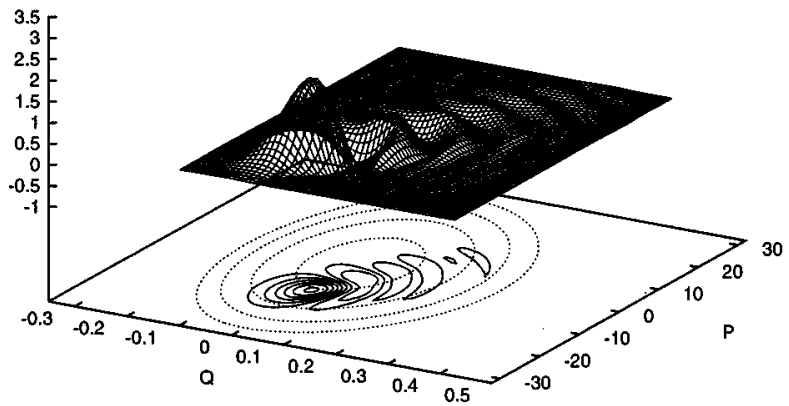

(e)

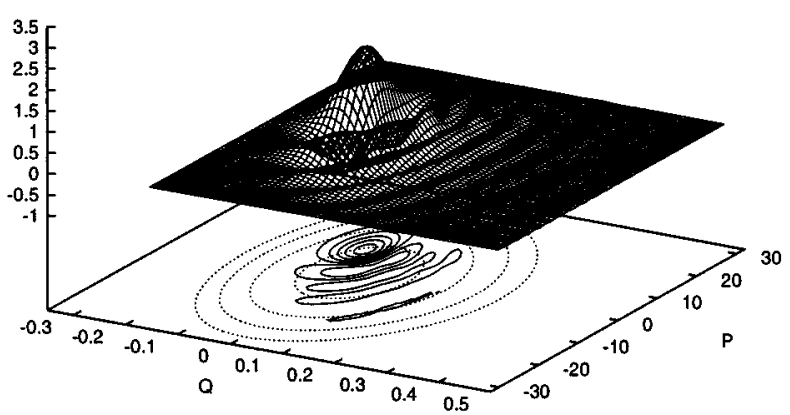

(g)

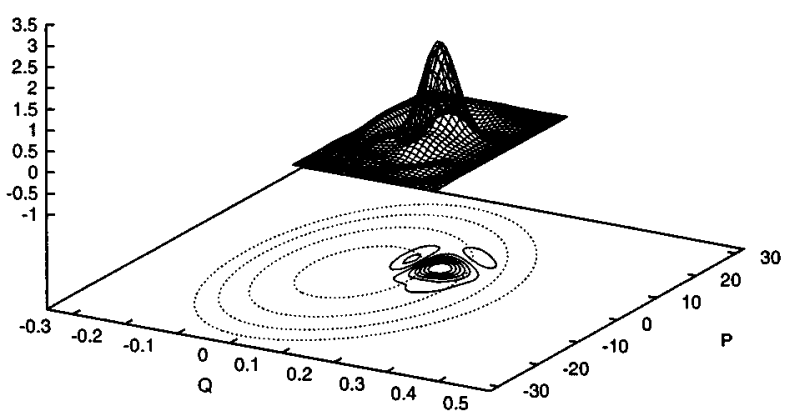

(b)

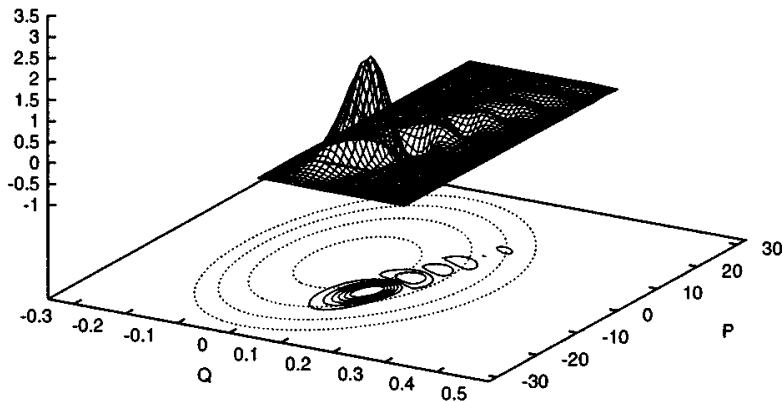

(d)

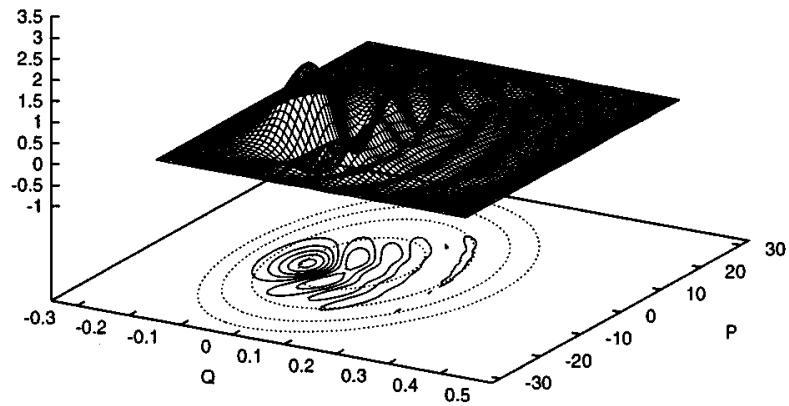

(f)

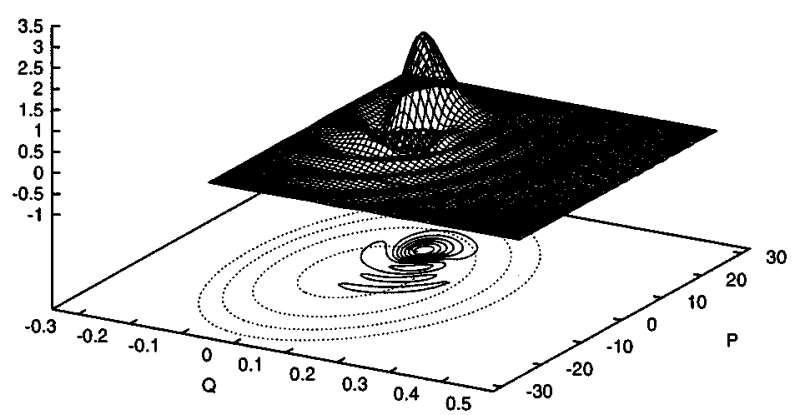

(h)

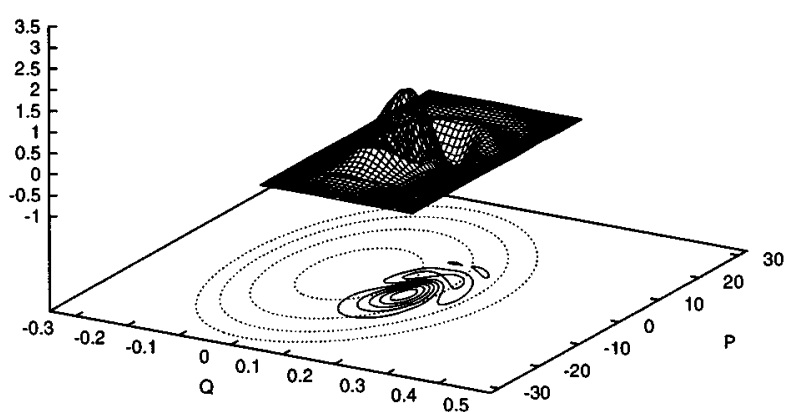

FIG. 5. (a),(b),..,(h) corresponds to the squeezed Gaussian being propagated in a Morse potential with $\lambda=120$, up to, respectively, $\tau=0$, $60, \ldots, 420$. Contours for the potential are taken as the first four energy eigenvalues, contours for the Wigner function are $-0.4,-0.1, \ldots, 1.7$.

$\rho(\mathbf{x})=J\left(p_{N}-p_{N-1}, q_{N}\right) \cdots J\left(p_{1}-p_{0}, q_{1}\right) f_{\psi}\left(q_{0}, p_{0}, 0\right)$.

Hence,

$$
\int d \mathbf{x} \rho(\mathbf{x})=\int d q_{N} \int d p_{N} f_{\psi}\left(q_{N}, p_{N}, t\right)=1 .
$$

However, since $\rho(\mathbf{x})$ is not positive definite, we cannot use it directly as sampling function. The trick needed, we get from the Metropolis sampling scheme; in this sampling scheme only a function proportional to the sampling function needs to be known, i.e., we can write Eq. (42) as 


$$
\begin{aligned}
P_{\phi}(t) & =2 \pi \hbar \int d \mathbf{x} f_{\phi}\left(q_{N}, p_{N}\right) \rho(\mathbf{x}) \\
& =2 \pi \hbar \frac{\int d \mathbf{x} f_{\phi}\left(q_{N}, p_{N}\right) \rho(\mathbf{x})}{\int d \mathbf{x} \rho(\mathbf{x})} \\
& =2 \pi \hbar \frac{\int d \mathbf{x} f_{\phi}\left(q_{N}, p_{N}\right) \operatorname{sign}[\rho(\mathbf{x})]|\rho(\mathbf{x})|}{\int d \mathbf{x} \operatorname{sign}[\rho(\mathbf{x})]|\rho(\mathbf{x})|}
\end{aligned}
$$

and sample after $|\rho(\mathbf{x})|$ as if its norm was unity.

This choice of sampling function is indeed a very good choice; it differs from the best, the norm of the integrand, by only $\left|f_{\phi}\left(q_{N}, p_{N}\right)\right| .^{12}$ Hence almost every variable is sampled the optimal, and that is without introducing any external knowledge of the system what so ever.

To test the scheme we propagated the ground state of a Morse oscillator and measured the overlap back on the ground state, i.e., we made a calculation of the quantity

$$
\begin{aligned}
1= & 2 \pi \hbar \int d q_{N} \int d p_{N} \int d p_{N-1} \cdots \\
& \times \int d p_{0} f_{0}\left(q_{N}, p_{N}\right) J\left(p_{N}-p_{N-1}, q_{N}\right) \cdots \\
& \times J\left(p_{1}-p_{0}, q_{1}\right) f_{0}\left(q_{0}, p_{0}\right),
\end{aligned}
$$

where $f_{0}$ is the Wigner function for the ground state of the Morse oscillator. ${ }^{10}$ We used for the calculation the same dimensionless variables as we used for the finite grid scheme, with $\tau=30$ and $N=60$, and were able to get the result to within a few percent. However, as always in Monte Carlo integration we were troubled with an enormous computation time compared to the finite grid scheme for a problem in a few dimensions. In the Monte Carlo method the bottleneck is the computation of the sampling function, which required three calls of the propagator function, which again is pretty hard to compute in its present form. In order to make a fair test of the performance of the present scheme we need a simpler expression for the short-time propagator. It should finally be pointed out that time propagation via Monte Carlo integration is only advantageous for high dimensional systems.

\section{CONCLUSION}

We asked the question: What can we gain by introducing a propagator in Wigner's phase space? We tried to answer the question by an example, namely the Morse oscillator. For this system we evaluated the short-time propagator, and used it in a new scheme to propagate a Gaussian distribution. The result of this calculation showed that this scheme is indeed an appropriate way to propagate directly in Wigner's phase space. We then used this new scheme to propagate classical distributions, and we saw that the classical behavior breaks down immediately as we start the propagation. However, as expected, if we make the potential almost harmonic the classical behavior is recovered.

An interesting perspective is the use of the phase space propagator in a Monte Carlo calculation. In this way we can avoid the introduction of some external sampling function, by simply using the norm of the propagator as a part of the sampling function. This should guarantee fast convergence, since this sampling function is the almost optimal choice.

Thus, this work might have interesting perspectives for the computational approach to quantum dynamics in systems of high dimensionality. To that end we have, however, at this early stage of the development one major unsolved problem-the evaluation of the short-time phase space propagator for general potentials in a form which is much simpler to evaluate then the short-time propagator considered in this paper. To be more specific, the remaining issue is how to evaluate the potential part of Eq. (16) for general potentials and how to do this in a way which leads to an expression which can be evaluated numerically in an inexpensive way.

\section{ACKNOWLEDGMENTS}

This work has been supported by the Danish Natural Science Research Council.

${ }^{1}$ N. Makri, Comp. Phys. Commun. 63, 389 (1991).

${ }^{2}$ E. P. Wigner, Phys. Rev. 40, 749 (1932).

${ }^{3}$ M. Hillery, R. F. O'Connell, M. O. Scully, and E. P. Wigner, Phys. Rep. 106, 121 (1984).

${ }^{4}$ F. McLafferty, J. Chem. Phys. 78, 3253 (1983).

${ }^{5}$ J. E. Moyal, Proc. Cambridge Philos. Soc. 45, 99 (1949).

${ }^{6}$ E. J. Heller, J. Chem. Phys. 65, 1289 (1976).

${ }^{7}$ N. E. Henriksen, G. D. Billing, and F. Y. Hansen, Chem. Phys. Lett. 149, 397 (1988).

${ }^{8}$ K. B. Møller, J. P. Dahl, and N. E. Henriksen, J. Phys. Chem. 98, 3272 (1994).

${ }^{9}$ M. Abramowitz and I. A. Stegun, Handbook of Mathematical Functions (Dover, New York, 1964).

${ }^{10}$ J. P. Dahl and M. Springborg, J. Chem. Phys. 88, 4535 (1988).

${ }^{11}$ R. Sala, S. Brouard, and J. G. Muga, J. Chem. Phys. 99, 2708 (1993).

${ }^{12}$ M. H. Kalos and P. A. Whitlock, Monte Carlo Methods (Wiley, New York, 1986).

${ }^{13}$ N. Metropolis, A. W. Rosenbluth, M. N. Rosenbluth, A. H. Teller, and E. Teller, J. Chem. Phys. 21, 1087 (1953) 